\title{
Naxos disease
}

INSERM

\section{Source}

INSERM. (1999). Orphanet: an online rare disease and orphan drug data base. Naxos

disease. ORPHA:34217

A recessively inherited condition with arrhythmogenic right ventricular

dysplasia/cardiomyopathy (ARVD/C) and a cutaneous phenotype, characterised by peculiar woolly hair and palmoplantar keratoderma. 